\title{
Entre lo que se dice y lo que se calla: visitando el concepto de empatía en la atención integral a la salud del adulto mayor
}

\section{Between what is said and what is silenced: visiting the concept of empathy in comprehensive health care for older adults}

\author{
Roberth Steven Gutiérrez-Murillo ${ }^{1, *}$, Walfrido Kühl Svoboda ${ }^{2}$, Carmen Justina Gamarra ${ }^{3}$, Érica \\ Ferreira de Souza ${ }^{4}$
}

1 Universidad Europea del Atlántico; MSc en Gerontología, España. Universidad Federal de la Integración Latinoamericana; Programa de Residencia Multiprofesional (Especialización) en Salud Familiar, Brasil; stevengumu@gmail.com, ORCID ID: 0000-0003-2304-3241

2 Universidad Federal de la Integración Latinoamericana; Profesor Adjunto al Curso de Grado en Salud Colectiva. Coordinador Académico del Programa de Residencia Multiprofesional en Salud Familiar. PhD en Ciencia Animal - Universidade Estadual de Londrina, Brasil; walfrido.svoboda@unila.edu.br, ORCID ID: 0000-0001-6320-4754

3 Universidad Federal de la Integración Latinoamericana; Profesora Adjunta al Curso de Grado en Salud Colectiva. PhD en Salud Colectiva - Universidade do Estado do Rio de Janeiro, Brasil; carmen.gamarra@unila.edu.br, ORCID ID: 0000-0003-4029-3859

4 Secretaría Municipal de Salud de Foz do Iguaçu, Paraná; Responsable Técnica de la Sección de Enfermedades y Agravios No Transmisibles. MSc en Salud Pública Basada en Evidencias - Universidade Federal de Pelotas, Brasil; ericafesil@hotmail.com, ORCID ID: 0000-0002-5425-145X

* Correspondencia: Avenida Brodoski 1095, Vila A, Foz do Iguaçu, Paraná, Brasil. CEP: 85869-050. E-mail: stevengumu@gmail.com

Recibido: 23/1/2021; Aceptado: 23/3/2021; Publicado: 26/3/2021

Resumen: La atención integral a la salud del adulto mayor es considerada una de las tareas más dinámicas y complejas para los sistemas públicos de salud y de asistencia social. Esta revisión narrativa constituye un ensayo crítico-analítico, de reflexión gerontológica sobre la práctica empática en la producción de actos de salud direccionados a resolver los problemas socio-sanitarios del segmento poblacional envejecido. Con tal visión, se propone una comprensión conceptual y práctica de la empatía, a través de la identificación de sus potencialidades y limitaciones en el contexto de la clínica geriátrica-gerontológica.

Palabras clave: ancianos; comunicación y educación en salud; envejecimiento; integralidad.

\begin{abstract}
Comprehensive health care for older adults is considered one of the most dynamic and complex tasks for public health and social care systems. This narrative review constitutes a criticalanalytical essay of gerontological reflection on the empathic practice in the production of health acts aimed at solving the socio-health problems of the aging population segment. With such view, a conceptual and practical understanding of empathy is proposed, through the identification of its potentialities and limitations in the context of the geriatric-gerontological clinic.
\end{abstract}

Keywords: aging; communication and health education; comprehensiveness; older adults.

\section{Introducción}

En el último siglo, la humanidad experimentó un giro existencial que desencadenó una serie de mudanzas estructurales notorias en todos los países del globo terrestre. Fenómenos tales como la industrialización, la tecnificación de las funciones laborales, el perfeccionamiento del saber científico y el aumento del grado de certeza en las prácticas médicas, sirvieron como punto de partida para el progreso contemporáneo. Sin embargo, 
referidos avances trajeron consigo una cuestión de observancia para la salud colectiva. Las sociedades hodiernas, ahora desarrolladas, se deparan con el contratiempo de repensar sus mecanismos de estructuración social, pese al aumento exponencial de individuos que alcanzan edades superiores a los 65 años. Se trata de los efectos secundarios del fenómeno social de envejecimiento poblacional, entendido como la explosión demográfica [babyboom] de los años 40 y 60, la combinación de la disminución de las tasas de fertilidad, la disminución de las tasas de mortalidad y el aumento de la esperanza de vida (1). Con una evidente pirámide etaria inversa, se expresa la necesidad de proporcionar una atención sanitaria integral a los ciudadanos envejecidos, contemplando los elevados índices de afección crónica, dependencia funcional y farmacológica, prevalentes en la vejez (2).

Dado que la atención integral a la salud del adulto mayor es considerada una de las tareas más dinámicas y complejas para los sistemas públicos de salud y de asistencia social, es esencial defender una línea de cuidados para el adulto mayor que presente eficiencia y eficacia, lo que requiere de una red asistencial articulada, referenciada y con un sistema de información diseñado sobre esa perspectiva (3). La Red de Atención a la Salud del Adulto Mayor [RASAM) envuelve todos los centros/institutos socio-sanitarios, públicos e/o privados, incumbidos por la prestación asistencial al segmento poblacional envejecido. Las RASAM, asimismo, se encuentran organizadas en consonancia con el Modelo de Gestión Sanitaria adoptado en cada país, mas éstas poseen generalmente mayor actuación en el campo de la atención primaria (4).

La complejidad en la asistencia al adulto mayor se da por múltiples factores, no estando relacionados obligatoriamente a los aspectos fisiopatológicos del envejecimiento natural. La premisa anterior encuentra sustento en la afirmación de que no existe una receta que sirva, de forma universal, para resolver todas las demandas del adulto mayor. Eso se cumple justamente por ser el envejecimiento humano un proceso heterogéneo, subjetivo e individual, debiendo ser observado a través de los lentes biológico, social, económico, político y ambiental $(3,4)$. Cabe de igual manera entender que la producción de actos en salud ${ }^{1}$, específicamente en el área geriátrica-gerontológica, posee un diferencial que merece ser destacado (5).

Se discute la falencia de las RASAM en adecuar los programas y servicios a los contextos socio-sanitarios que configuran la realidad existencial en la que los ancianos se encuentran inseridos. Con todo, el problema central radica en el desacierto de enfocar las intervenciones (clínicas o no) en la manifestación patológica, antes de proyectar al adulto mayor como un ser holístico. Desde ese momento, puede considerarse que existe una ruptura del contacto integral, pues se ignora la posibilidad de ofrecer una atención plena y humanizada. Tales comportamientos surgen, en la mayoría de las veces, por una formación académica basada en paradigmas asistenciales hegemónicos poco compatibles con los tiempos actuales, reflejando una práxis que no consigue responder ni acompañar, de manera cierta, a las exigencias socio-sanitarias del adulto mayor moderno.

Investigaciones reiteran que el modelo tradicional de atención centrado en la enfermedad, la curación y la rehabilitación, ofrece medidas desproporcionadas y costosas para los servicios de salud, además de que no tiene en cuenta el sufrimiento del paciente y

1 En este texto los autores adoptan la definición de 'actos en salud' propuesta por Emerson E. Merhy (1994), que afirma que "todos: los trabajadores, los usuarios y los gestores de los servicios saben también que, para alcanzar esos fines, el conjunto de actos produce un determinado formato de atención, de diferentes maneras: como actos de acciones individuales y colectivas y como planteamientos clínicos y sanitarios de los problemas de salud; combinan todos los conocimientos y prácticas que intervienen en la construcción de los actos de atención y conforman los modelos de atención sanitaria" (Traducción libre del portugués, p. 1). 
sus familiares, omitiendo el manejo integral de los síntomas y malestares (6). Lo anterior conlleva a pensar que la atención debe organizarse de forma integrada y los cuidados deben coordinarse a lo largo de la ruta asistencial, en una lógica de red desde la entrada en el sistema hasta el final del cuidado de la vida (3).

Recientemente, la empatía ha sido un tema de interés para los profesionales de diferentes áreas del conocimiento, desempeñando un papel importante en el desarrollo de las habilidades sociales, cognitivas y afectivas (7). Un concepto inicial de empatía en el ámbito sanitario podría ser dado a la capacidad del profesional para ponerse en el lugar del usuario/paciente y poder, así, comprender su punto de vista, sus reacciones y sentimientos frente a su estado de salud (8).

En el presente trabajo, la práctica empática como herramienta para promover la atención integral al geronte es tomada como objeto de reflexión. El texto privilegia el discurso gerontológico que defiende la concepción de la salud biopsicosocial en la vejez. De tal modo, objetivase proponer una comprensión conceptual y práctica de la empatía en el contexto de la clínica geriátrica-gerontológica, a través de la identificación de sus potencialidades y limitaciones. Por lo tanto, se optó por realizar una revisión narrativa no exhaustiva de ensayos que defienden, a partir de diversas perspectivas, la relevancia de su apropiación en la producción de actos en salud en el contexto de la clínica geriátricagerontológica. Así siendo, fue realizada una consulta virtual a las bases de datos científicos: Google Académico, Taylor \& Francis Online (periódicos especializados en geriatría y/o gerontología) y Scientific Electronic Library Online - SciELO, empleando los descriptores 'adulto mayor' + 'empatía'; 'anciano' + 'empatía' + 'práctica empática'; 'elderly' + 'empathy'; 'older adults' + 'empathy'; 'idoso' + 'empatia', sin exclusión temporal. Se seleccionaron los trabajos que contemplaron intervenciones con profesionales sanitarios y/ o adultos mayores en el adviento de la práctica empática. Fueron extraídas las principales informaciones/observaciones, respetando la originalidad de los autores y presentadas de forma comparativa, lo que permitió identificar similitudes y contrastes en la literatura.

Estructuralmente, el texto ha sido dividido en cuatro partes. De comienzo, se abraza una breve contextualización histórico-conceptual, principalmente con el interés de entender el surgimiento del término 'empatía' y su relación con el campo sanitario. A su vez, continúa un espacio crítico-descriptivo que recaba diferencias entre los conceptos de 'empatía' y 'simpatía', tan poco claros en la labor sanitaria geriátrica y gerontológica. En un tercer momento, se pondera el empleo de la práctica empática como una herramienta multidimensional capaz de promover la atención integral de los usuarios envejecidos. Como punto de clausura, el trabajo extiende una revisión argumentativa sobre las posibilidades y limitaciones de adoptar la práctica empática en el ambiente de la clínica geriátrica-gerontológica.

\section{Entendiendo el concepto y origen de empatía}

Preliminarmente, fue realizada consulta on-line al Diccionario Médico-biológico, Histórico y Etimológico [Dicciomed, Universidad de Salamanca], para lo que se extrajo el siguiente resultado a la palabra 'empatía': capacidad para participar en los sentimientos de otra persona y asimilarse a su estado anímico; nace de la identificación con otro y la comprensión íntima de su situación existencial (9).

En 1986, fue publicado un importante trabajo que tuvo por finalidad aclarar y rehabilitar la utilización del concepto de empatía, a partir de la década de 1900 (10). En éste, se admitió que el término 'empatía' es comparativamente reciente, habiendo sido acuñado por Titchener (1909), en 'Psicología Elemental de los Procesos del Pensamiento' (Del Inglés 
Elementary Psychology of the Thought Processes). Titchener propuso la traducción de la palabra 'Einfühlun' del alemán, para 'empathy' al inglés, utilizada cuando las personas se proyectan sobre los objetos de percepción y en ese proceso ocurre el desdibujamiento de la distinción entre 'el yo' y 'el objeto' (11).

San-Martín y Ortega-Sánchez (12) retoman discusión en relación al origen etimológico. Comentan que éste puede intuirse en el ámbito del pensamiento griego. Toman como referencia 'Arte Poética', de Aristóteles, entrelazándolo con las concepciones de piedad y compasión ['ElEos] siendo componentes emocionales de la tragedia (12). Sin embargo, es a partir de los estudios de la Psicología Social que se propaga su uso en otros campos del conocimiento sanitario. Inicialmente, empatía fue entendida como el intento de 'un yo consciente' de comprender sin juzgar las experiencias positivas y negativas de 'otro yo' y estaba fuertemente vinculada a la idea de captar los sentimientos de los otros, con los propios músculos de la mente [Empatheia] (10).

A pesar de los varios esfuerzos por ofrecer una explicación al concepto de empatía, cuatro enfoques diferentes prevalecieron: el condicionamiento vicario; las atribuciones diferenciales; la ayuda empática y la capacidad empática (10). Para el condicionamiento vicario, el punto clave era que la observación de las acciones y las respuestas emocionales de otros contribuye a la propagación del comportamiento y la emoción de un individuo a otro (13). En el caso de las atribuciones diferenciales los actores hacen más atribuciones situacionales mientras que los observadores ofrecen más explicaciones de disposición (10, 11).

Los últimos dos enfoques comparten ciertas características, aunque sufren de divergencias en la práctica. La ayuda empática sería una variable dependiente arreglando la situación experimental, de manera que cualquier ayuda que ocurriera pudiera ser considerada desde algún punto de vista empático (10). Finalmente, la capacidad empática, que corresponde al conjunto de habilidades psicosociales relevantes para la resolución de problemas del otro, requiriendo una dosis de comprensión propia, pero que no consiste obligatoriamente en su concordancia (14).

Con el pasar del tiempo y, por efecto de la comprobación hipotética, nuevas significaciones han emergido. Pensadores contemporáneos deambulan entre las eminentes vertientes psicológicas, posibilitando nuevos ideales que permitan una mejor comprensión de la práctica empática en el contexto del cuidado a la salud. Particularmente, las mudanzas conceptuales admitidas históricamente han servido de base para adquirir pensamientos más precisos sobre las relaciones de cuidado < paciente - profesional sanitario >

Las contribuciones de Mark H. Davis (1980) pincelaron importantes avances para el estudio de las relaciones sociales, incluyendo aquí la empatía. Este tomó interés en demostrar que, aunque escalas pertinentes para medir comportamientos empáticos ya habían sido implementadas años atrás, éstas carecían de un importante factor: el desarrollo de una medida de empatía individual de diferencias multidimensionales (15). Su fundamento abrazó que "los componentes cognitivos y afectivos de la empatía comprenden un sistema interdependiente en el que cada uno influye en el otro", motivo por el cual estos "nunca podrán entenderse plenamente mientras los esfuerzos de investigación se concentren en un aspecto de la exclusión relativa del otro" (15).

Años más tarde, Davis (1983) culminó sus observaciones por medio de la publicación de un instrumento ampliado, al cual llamó de Índice de Reactividad Interpersonal - IRI (Interpersonal Reactivity Index, del inglés), ahora entendiendo que la empatía, en el sentido más amplio, "se refiere a las reacciones de un individuo ante las experiencias observadas de 
otro" (16). La composición estructural del IRI contiene cuatro elementos basilares, proyectados paralelamente como subniveles, a saber: toma de perspectiva; fantasía; preocupación empática y angustia personal. Mencionado instrumento ha sido ampliamente aceptado por los estudiosos a nivel mundial y suele ser aprovechado en la conducción de intervenciones exploratorias en los ramos de la Psicología Social y en la Psicología Clínica Comportamental.

Edith Stein (1922), por su parte, ofreció observaciones sobre la fenomenología sensual y emocional de la empatía. Anclada sobre las mismas bases conceptuales de Titchener (1909), la autora se centra en comprobar que los sentimientos no operan sólo a un nivel de profundidad sensual o emocional; también se pueden distinguir en cuanto a duración, alcance e intensidad. Más precisamente, Stein "defiende la línea divisoria entre los sentimientos que sólo tienen una cualidad experiencial y los sentimientos que también tienen un contenido de significado al estar sobre las cosas en el mundo" (17). Con su auxilio teórico, es posible aclarar que empatía no significa imitar los sentimientos ajenos, mas adquirir un sentido de comprensión del 'otro yo' (17).

Muchas son las formas de pensar y entender la empatía. Sirve notar que su entendimiento se relaciona directamente con la visión de mundo y las características intrínsecas de los participantes en ese proceso <quien es empatizado y quien concluye el acto empático >. Toro-lópez y colaboradores (2020) refuerzan tal enunciado, al avalar que "la empatía cambia con las características de los empatizadores y las situaciones, estas dos características se pueden conciliar cuando reconocemos el papel clave de la motivación para impulsar a las personas a evitar o abordar el compromiso con las emociones de los demás" (18).

\section{Ser empático no significa ser simpático: empatía $\neq$ simpatía}

Es común, en el día a día de las personas, avistar ocasiones en las que el uso de los conceptos de empatía y simpatía se presenta en la forma de sinónimo, eso se vuelve aún más habitual en la práctica clínica. Dicha costumbre, empero, debe ser identificada e instruida inmediatamente, pues ambos términos poseen finalidades opuestas. Lejos de querer hacer exhaustiva tal diferenciación <empatía $\neq$ simpatía>, para fines propios del presente texto se admite el pensamiento de Wispé (1986) que distingue 'simpatía' como la mayor conciencia del sufrimiento de otra persona como algo que debe ser aliviado y 'empatía' como el intento de un yo consciente de comprender sin juzgar las experiencias positivas y negativas de otro yo.

En tono razonable, Wispé (1896) llama la atención para el uso de la simpatía y su inconveniencia en la práctica clínica, en la cual se considera el consecuente cuidado al adulto mayor (10). Veamos que:

La simpatía no facilita las evaluaciones precisas. No se puede ser comprensivo y objetivo. La simpatía se presta a las emociones distorsionadas. Esta posibilidad podría probarse atributivamente en el laboratorio y en un sentido más práctico por las historias personales. La simpatía tampoco es el modo de interacción terapéutica. Simpatía puede llevar a una identificación emocional más cercana y a una identificación perentoria de acciones de rescate en nombre del paciente. También puede conducir a una versión romántica de la neurosis o locura del paciente como el único recurso en un mundo loco. La comprensión compasiva es una cosa en la terapia; la simpatía es otra (Traducción libre del inglés, p. 319). 
Por esa razón, importa comprender que el acto de ser empático no debe relacionarse con la adquisición de comportamientos egocéntricos o insensibles. En su esencia, la práctica empática demanda de una conducta humanizada e integral, en lo que respectan los apuros de la clínica geriátrica-gerontológica, pero no admite una compasión generalizada, como se infiere en el caso de la simpatía. De ese modo, en la empatía 'el yo' es el vehículo para la comprensión, y nunca pierde su propia identidad (10).

Desde la perspectiva de género, la literatura científica ha demostrado que la práctica empática acostumbra ser más prevalente en individuos del sexo femenino (19). Generalmente (con variaciones expresivas por mayor edad), las mujeres parecen tener más facilidad para responder de forma empática al cuidado geriátrico, posiblemente por influencia de los comportamientos sociales a ellas demandados. En efecto, el cuidado en salud del adulto mayor ha sido históricamente prevalente en la población femenina, principalmente en el ambiente domiciliar y en las instituciones filantrópicas donde predomina su presencia. Estudios en el campo de la neurociencia social también vienen preocupándose por ofrecer posibles respuestas al nivel de activación cerebral, investigando correlaciones entre el género y el hemisferio derecho, advirtiendo mayor respuesta en el sexo femenino a estímulos empáticos $(19,20)$.

Las manifestaciones empáticas constituyen una respuesta positiva y de apoyo a la situación vivenciada. La empatía, conforme explicado por Chismar (1988), permite que la persona <tomemos aquí el caso del profesional sanitario> sienta lo que el adulto mayor está sintiendo, pero no implica el tipo de reciprocidad y sentimiento de 'compañerismo' envuelto en la simpatía (21). En consecuencia, para que el profesional sanitario experimente un posicionamiento empático en su quehacer con ancianos, este debe, al menos de alguna manera, preocuparse o interesarse por el destinatario <tomemos aquí el caso del adulto mayor $>$. No obstante, la práctica empática no vendría a determinar una preocupación extendida o duradera, más bien inmediata o fugaz. De ese modo, el profesional sanitario es estimulado, perturbado o incluso conmovido por el estado de salud del destinatario (21).

Los enfoques asistenciales empáticos son más deseables que los simpáticos en el contexto de la clínica geriátrica-gerontológica, una vez que estos últimos conducen a la elevada excitación emocional por parte del profesional sanitario, alimentando fuertes sentimientos individuales hacia el adulto mayor. Particularmente, este es un aspecto que merece especial atención. Como ya dialogado en la sección 2, las atribuciones diferenciales permiten al observador <profesional sanitario> instituir explicaciones de disposición frente a la situación del adulto mayor, éstas a su vez poseen un potencial desviador, en la medida en que se encuentran ampliamente sugestionadas por los procesos de orientación emocional, en que juegan papeles selectivos los sentimientos de tristeza, soledad, compasión y piedad.

En la otra cara de la moneda, acepciones que se sobrepongan a los sentimientos supramencionados necesitan ser observadas con moderación, pues se admite que ambas emociones, positivas y negativas, cumplen funciones sui generis con igual grado de importancia para el adulto mayor (23). Es también relevante saber que una comprensión equívoca sobre los fundamentos de la empatía clínica podría levantar bandera roja para el riesgo de fijar un carácter apático, frío y poco profesional, impidiendo el goce de una real atención integral en la vejez (22). 


\section{La empatía como herramienta para promover la integralidad del adulto mayor}

Entender, practicar y evaluar la empatía en los servicios de salud se vuelve una herramienta fundamental para los profesionales sanitarios, puesto que constituye una competencia interprofesional que les permite vincularse afectivamente con el adulto mayor $(7,16)$. Cabe señalar que no es una tarea únicamente sanitaria, como podría cogitarse desde una revisión de origen. Se ha vuelto, más bien, una actividad multiprofesional benéfica tanto para el adulto mayor (que recibe un cuidado más asertivo) y para el profesional (que conquista nuevas habilidades socioemocionales).

Queda claro que la empatía hace parte de las funciones comunico-educativas en salud. Por un lado, es una acción de dependencia comunicativa, ya que se deriva de los elementos básicos de la comunicación asertiva. Y, por otro, es una acción de educación sanitaria ya que la transmisión de información a individuos y a grupos mediante la comunicación social crea el conocimiento que sirve de base para lograr los cambios de actitudes y prácticas saludables (24). Del trabajo de Hernández (2011) es posible rescatar que: 1) "el comportamiento se considera mediado por el conocimiento, es decir, lo que sabemos y lo que creemos afecta a la manera como actuamos" y que 2) "el conocimiento es necesario, pero no suficiente para producir cambio de comportamiento. También desempeñan funciones importantes las percepciones, los sentimientos, la motivación, las aptitudes y los factores del entorno social". Tales apuntamientos adquieren una intensa connotación en el trabajo comunico-educativo en salud del adulto mayor (25).

No obstante, es innegable que el ser humano carece de destrezas empáticas al nacer. Por ello, éstas precisan ser aprendidas, incorporadas y validadas, justo como cualquier otra aptitud de cuño social. Con esa misma lógica, Muñiz (2018) referenciando Monjas (2011), alerta que "no existe una definición consensuada de competencia social pues se trata de un constructo que engloba diversas habilidades integradas al servicio de metas personales concretas, sabiendo cómo aplicarlas en cada contexto y situación interpersonal" (26).

Tal posicionamiento permite inferir que, para que la práctica empática sea competente, esta debe efectuarse en situaciones conducentes generalmente imprevisibles en el quehacer sanitario. Es un punto certero, pues se sabe que todo atendimiento/consulta que demande respuestas inmediatas o paliativas a los menesteres del adulto mayor, requiere de una alta capacidad observacional y compromiso ético, además del conocimiento sanitario [atributos específicos de cada profesión].

Es en dicho contexto que la práctica empática encuentra su propósito y aplicabilidad, pues se convierte en una cualidad deseable para la producción de los actos en salud. Se trata de una competencia laboral blanda, que sujeta rasgos de la personalidad del propio profesional sanitario. Esa respuesta puede darse en situaciones identificadas por el adulto mayor como negativas, o bien, en aquellas en las cuales hay manifestación de emociones satisfactorias. Lo importante es, absolutamente, percibir las causas de tales emociones sin perder la noción territorial de 'el yo' y el 'otro yo' (27).

$\mathrm{Al}$ afirmar que el camino para el cuidado integral al adulto mayor parece aún no estar claro para los profesionales sanitarios, los gerentes y para el propio usuario de los sistemas de salud, Guedes y colaboradores (2017) tensionan una cuestión gerontológica contemporánea (28). Admiten que el apoyo social se encuadra como pieza esencial de la atención integral en salud del adulto mayor. Aquí, lo central está en captar la potencialidad articuladora de las redes sociales (considerando las esferas de prevención, 
promoción, rehabilitación y curación), reteniendo conjuntamente que las características de esas redes pueden variar en función de los papeles adoptados por instituciones públicas y privadas (gobernanza), por parientes (núcleo y estructura familiar) y por amigos y vecinos (vínculo comunitario).

Medeiros y colaboradores (2017) se dan a la tarea de destacar que la integralidad en la atención al adulto mayor, dentro de la atención primaria en salud, necesita incorporar una visión más amplia del individuo. Es vital llamar la atención de los profesionales sobre la importancia de observar los aspectos cognitivos, el humor, la movilidad y la comunicación como dominios esenciales para la salud, ampliando el ámbito de su acción más allá de las clásicas enfermedades crónicas (29). Aún así, esta producción de actos en salud integrales, asertivos y con potencial de mudanza se encuentra estrictamente limitada a la existencia del vínculo entre el adulto mayor y el profesional sanitario. Este tipo de relación asistencial, si bien tornaría el cuidado más íntegro y pleno, muchas veces no es conquistado en la actividad sanitaria, justamente por la alta cobranza <en términos de consultas/atendimientos médicos $>$, y demandas reprimidas en el sistema público de salud, que acaban por apresar el contacto entre ambas partes $\mathrm{y}$, con eso, incorporar barreras socio-afectivas.

Valorando el factor ventajoso de fomentar un vínculo asistencial con el adulto mayor, Seixas y colaboradores (2019) resaltan que "en el encuentro con el otro es que podemos percibir que necesitamos de él para construir buenas salidas" (30). Con tal característica, postulan que en ese encuentro es posible depararse tanto con usuarios <adultos mayores> rebeldes que carecen de interés en el proyecto asistencial que les es ofrecido, tantos usuarios idealizados, con un perfil común, sumiso, que fácilmente irán aceptar las proposiciones colocadas. Con base en lo anterior, el planteamiento del profesional sanitario debe ser rigorosamente político, buscando siempre los mayores beneficios para sí. Atinan, por cierto, al decir que "cuando el otro está en busca de ayuda, ya trae consigo, entre líneas, una propuesta, pero pocas veces nos detenemos a escucharla, y no solemos preguntar: ¿Qué te gustaría que hiciéramos para ayudarte?" (30).

\section{La práctica empática en la clínica geriátrica-gerontológica: posibilidades y limitaciones}

El cuidado a la salud del adulto mayor designa intervenciones de naturaleza multidimensional. Es, indudablemente, un dominio asistencial desafiador que aumenta su grado de complejidad conforme las estructuras societarias mudan e incorporan nuevas tecnologías en la práxis sanitaria. Por eso, Yuguero y colaboradores (2018) aclaran que "la práctica clínica de calidad no incluye tan solo la mejora de conocimientos en aspectos científicos y técnicos, sino también en la gestión de valores, la sensibilidad ética y las habilidades en la valoración y manejo de los conflictos éticos" (31).

Uno de los principales obstáculos para adoptar la práctica empática en el contexto de la clínica geriátrica-gerontológica <especialmente en el cuidado paliativo o psiquiátrico>, es la posibilidad de que los profesionales sanitarios puedan sufrir fatiga/estrés por compasión. Para García (2017), se trata de "una respuesta ante el sufrimiento de un individuo más que a la situación laboral" (32), en complemento, Albaladejo (2019) la define como el "coste de cuidar a otros que sufren dolor emocional" (33). Indiferentemente, tales comportamientos se extienden sobre las esferas física, emocional, social y espiritual, interfiriendo consecuentemente en la vida personal y profesional de los trabajadores sanitarios. 
En ese mismo ángulo aprensivo, Carrió (2018) advierte que "médicos y enfermeras, entre otras profesiones, tienen que saber manejar la distancia terapéutica para poder pensar con claridad lo mejor para cada paciente, pero sin caer en la frialdad del técnico. De manera concreta deben conocer los peligros que suponen las trampas de piedad" (34). Lo cierto es que, profesionales sanitarios situados en ambientes hospitalarios se tornan altamente propensos al contagio afectivo ${ }^{2}$ [Gefühlsansteckung], por el hecho de vivenciar constantemente el sufrimiento biopsicosocial del adulto mayor $\mathrm{y}$, en muchas ocasiones, el duelo de sus seres queridos (35).

De forma diferente, cuando la empatía es entendida y correctamente aplicada en escenarios oportunos, ésta puede resultar en la satisfacción por compasión, o sea, "la capacidad para recibir gratificación por dispensar cuidados, lo que es un aspecto positivo en el desempeño de la función del profesional sanitario" (35). Ídem, Pintado (2018) destaca la implementación de intervenciones en mindfulness, justamente por haber demostrado excelentes resultados para disminuir los índices de fatiga/estrés por compasión y aumentar la capacidad de auto-compasión (36).

Por ser una práctica dependiente del contacto social, cuando existe un vínculo estrecho entre el adulto mayor y el profesional sanitario, estos son más capaces de producir momentos empáticos. Eso ocurre porque la enfermedad/padecimiento del adulto mayor ya es de conocimiento del profesional sanitario, motivo por el cual el adulto mayor se coloca en una posición confortable para compartir informaciones más detalladas sobre su estado de salud (18). En esos ambientes, la producción de actos en salud podría verse fortalecida, pues hay ausencia del juzgamiento previo y escucha activa, al mismo tiempo, consideración de las autopercepciones del adulto mayor sobre su estado de salud y las experiencias propias del profesional sanitario, siendo elementos optimistas que favorecen la adopción de la práctica empática en el ámbito de la clínica geriátricagerontológica (37).

La tabla 1 sintetiza los beneficios de adoptar un atendimiento socio-sanitario empático con el adulto mayor, en relación con el tipo de intervención y las características intrínsecas de los individuos abordados, según consulta a la literatura gerontológica que aborda el estudio de las intervenciones empáticas. Véase:

Tabla 1: Caracterización de pesquisas que aluden posibilidades de la práctica empática en el contexto de la clínica geriátrica-gerontológica, 2021.

\begin{tabular}{|l|l|l|l|}
\hline $\begin{array}{l}\text { AUTOR(es), } \\
\text { AÑO }\end{array}$ & ORIGEN & PÚBLICO & BENEFICIOS OBSERVADOS/ESPERADOS \\
\hline $\begin{array}{l}\text { Monteiro, } \\
\begin{array}{l}\text { Maeirós \& } \\
2014 \text { (38). }\end{array}\end{array}$ & Portugal & $\begin{array}{l}78 \text { cuidadoras } \\
\text { formales de } \\
\text { AM* }\end{array}$ & $\begin{array}{l}\text { Ser empático (emocional y cognitivamente), aún en el contexto } \\
\text { de dependencia funcional avanzada del AM, constituyó factor } \\
\text { protector para Síndrome de Burnout. }\end{array}$ \\
\hline $\begin{array}{l}\text { Araújo \& } \\
\begin{array}{l}\text { Costa, 2016 } \\
(39) .\end{array}\end{array}$ & Brasil & $\begin{array}{l}23 \text { pares de } \\
\text { AM- } \\
\text { Acompañante }\end{array}$ & $\begin{array}{l}\text { El estado emocional del AM fue fácilmente identificado por su } \\
\text { acompañante. El vínculo entre AM-Acompañante ofrece la } \\
\text { capacidad de colocarse y simular la perspectiva subjetiva del } \\
\text { AM frente a sus desafíos sanitarios. }\end{array}$ \\
\hline $\begin{array}{l}\text { Gholamzade } \\
\text { h, } \\
\text { Khastavaneh, } \\
\text { Khademian, } \\
\text { et al, 2018 } \\
(40) .\end{array}$ & Irán & $\begin{array}{l}63 \text { estudiantes } \\
\text { de Enfermería }\end{array}$ & $\begin{array}{l}\text { La capacitación en aptitudes de empatía mejoró la empatía y } \\
\text { las actitudes de los estudiantes de enfermería hacia los AM. } \\
\text { Por lo tanto, se recomienda incorporar el entrenamiento de } \\
\text { empatía en el currículo de enfermería de pregrado. }\end{array}$ \\
\hline $\begin{array}{l}\text { Hannan, } \\
\text { Sanchez, }\end{array}$ & Estados & $\begin{array}{l}181 \\
\text { estudiantes }\end{array}$ & $\begin{array}{l}\text { La práctica empática de los médicos afectó significativamente } \\
\text { la percepción de los estudiantes sobre errores médicos, }\end{array}$ \\
\hline
\end{tabular}

2 Los autores toman como referencia el concepto adoptado por Max Scheler (1973), en su libro ‘Esencia y formas de simpatía'. 


\begin{tabular}{|c|c|c|c|}
\hline $\begin{array}{l}\text { Musser, et al, } \\
2019 \text { (41). }\end{array}$ & Unidos & $\begin{array}{l}\text { de Psicología } \\
\text { y Enfermería }\end{array}$ & validando el trabajo interprofesional previo. \\
\hline $\begin{array}{l}\text { Longhi, } 2015 \\
\text { (42). }\end{array}$ & Italia & $\begin{array}{l}40 \\
\text { profesionales } \\
\text { sanitarios de } \\
\text { un hospital } \\
\text { público }\end{array}$ & $\begin{array}{l}\text { El abordaje empático de las demandas del AM permitió al } \\
\text { personal sanitario identificar situaciones de abuso } \\
\text { multidimensional contra ancianos, no sólo en el terreno } \\
\text { hospitalario, mas también en el domiciliar. }\end{array}$ \\
\hline $\begin{array}{l}\text { Fuentes, } 2014 \\
\text { (43). }\end{array}$ & España & $\begin{array}{l}117 \text { AM } \\
\text { comunitarios }\end{array}$ & $\begin{array}{l}\text { Un adecuado nivel de apoyo social al AM recibido por } \\
\text { parientes (principalmente hijos/nietos), promueve } \\
\text { comportamientos autónomos y saludables, en las esferas } \\
\text { emocional e instrumental. La participación del AM en el } \\
\text { proceso de cuidado refuerza la práctica empática domiciliar. }\end{array}$ \\
\hline $\begin{array}{l}\text { García-Vera, } \\
\text { Robles- } \\
\text { Rodríguez, } \\
\text { Villegas- } \\
\text { Bernabé, et } \\
\text { al, } 2016(44) \text {. }\end{array}$ & México & $\begin{array}{l}\text { Médicos } \\
\text { especializado } \\
\text { s en Medicina } \\
\text { Familiar }\end{array}$ & $\begin{array}{l}\text { Para llevar a cabo una buena relación médico-paciente, el } \\
\text { médico familiar deberá mostrar interés por el paciente, utilizar } \\
\text { palabras comprensibles, proporcionar información sobre el } \\
\text { tratamiento, generar tranquilidad, aconsejar, pero sin influir en } \\
\text { la toma de decisiones, así como permitir que este exprese sus } \\
\text { puntos de vista, inconformidades, dudas y necesidades. }\end{array}$ \\
\hline $\begin{array}{l}\text { Pison, Yáñez, } \\
\text { Fritis, et al, } \\
2019 \text { (45). }\end{array}$ & Chile & $\begin{array}{l}191 \\
\text { estudiantes } \\
\text { de } \\
\text { Kinesiología }\end{array}$ & $\begin{array}{l}\text { Los autores recomiendan: cambios completos del currículo de } \\
\text { la carrera, cambios de enfoques de los métodos de enseñanza } \\
\text { (eminentemente activos); formalización del currículo oculto } \\
\text { positivo, el contacto temprano del estudiante con el paciente } \\
\text { en un ambiente de aprendizaje positivo, formación del docente } \\
\text { y de las autoridades administrativas de las universidades, los } \\
\text { cuales no pueden ser considerados como agregados en la tarea } \\
\text { de lograr el aumento sostenido y sistemático de los niveles de } \\
\text { empatía en los estudiantes. }\end{array}$ \\
\hline $\begin{array}{l}\text { Morales- } \\
\text { Concha, } \\
\text { Ccarita- } \\
\text { Yucra, } \\
\text { Marroquin- } \\
\text { Santa Cruz, } \\
\text { et al, } 2017 \\
(46) \text {. }\end{array}$ & Perú & $\begin{array}{l}361 \\
\text { estudiantes } \\
\text { de Medicina }\end{array}$ & $\begin{array}{l}\text { Estudiantes cursando el último y penúltimo año de la carrera } \\
\text { presentan mayores índices empáticos al contacto con los AM. } \\
\text { Esto se debe a la alta carga práctica (clínica ambulatoria y } \\
\text { especializada) y la vivencia del servicio comunitario. Trabajar } \\
\text { la empatía de forma temprana promueve la formación de } \\
\text { médicos humanizados y con convicciones asistenciales más } \\
\text { integrales. }\end{array}$ \\
\hline $\begin{array}{l}\text { San-Martín, } \\
\text { Roig-Carrera, } \\
\text { Villalonga- } \\
\text { Vadell, et al, } \\
2017 \text { (47). }\end{array}$ & España & $\begin{array}{l}156 \text { médicos } \\
\text { residentes }\end{array}$ & $\begin{array}{l}\text { Se ha demostrado la influencia de los factores sociales y } \\
\text { culturales en las diferencias observada en la orientación } \\
\text { empática entre profesionales sanitarios. Además de la empatía, } \\
\text { las habilidades de trabajo colaborativo interprofesional y de } \\
\text { aprendizaje médico permanente han sido reconocidos como } \\
\text { elementos esenciales de la excelencia profesional del médico. }\end{array}$ \\
\hline $\begin{array}{l}\text { Díaz- } \\
\text { Martínez, } \\
\text { Armesto \& } \\
\text { Rojas, } 2020 \\
(48)\end{array}$ & Colombia & $\begin{array}{l}\text { Estudiantes } \\
\text { de Medicina }\end{array}$ & $\begin{array}{l}\text { En el contexto de malas noticias (duelo/luto), un correcto } \\
\text { dominio empático de la situación fatídica por parte del médico } \\
\text { apaciguaría el impacto emocional por la pérdida del ser } \\
\text { querido, al mismo tiempo en que evitaría la sobrecarga } \\
\text { emocional de sí. }\end{array}$ \\
\hline
\end{tabular}

Fuente: Elaboración propia de los autores (2021). *AM: Adultos Mayores.

La competencia clínica geriátrica-gerontológica, definida por Martínez-Gonzáles y colaboradores (2020) como "una combinación de conocimientos, habilidades y actitudes para desempeñarse adecuadamente en un entorno de atención al paciente envejecido" se ve verdaderamente favorecida cuando existe la apropiación de la práctica empática por 
parte del personal sanitario (49). No sólo se anhela una atención completa y de calidad, sino que se reitera el compromiso social con los ciudadanos de mayor edad (Tabla 1).

A pesar de la gama de utilidades ya señaladas, la institucionalización de la práctica empática en los servicios de salud especializados en la atención al adulto mayor todavía incurre en cuantiosas injerencias ideológicas. Recalcar y discutir los entrabes institucionales que cohíben al adulto mayor de disfrutar de un atendimiento integral debe ser una actitud prevalente en la labor geriátrica-gerontológica, sirviendo como acreditativo vocacional para el profesional sanitario que opta por esa área del conocimiento. Ahora bien, esa no debe ser una misión exclusiva del recurso humano, pues lo mismo les corresponde a las RASAM públicas y/o privadas prestadoras de servicios socio-sanitarios a la población geronte. Ambas partes poseen un objetivo mutuo, cual sea contribuir con el dominio de un proceso (individual y colectivo) de envejecimiento saludable y autónomo, al lado de una elevada calidad de vida en la vejez.

La práctica empática ha sido relacionada con una visión constructiva de las relaciones socio-afectivas que pueden emerger en el nexo profesional sanitario - adulto mayor, por el efecto de entender la situación/contexto del otro yo $(22,31)$. Sin lugar a vacilaciones, esa perspectiva ha sido el hincapié para el desarrollo de sistemas asistenciales más exhaustivos y resolutivos. En compensación, número considerable de doctos se han embelesado por entender el lado 'negativo' o los efectos adversos de la empatía.

En Breithaupt (2018) la empatía es concebida como capacidad de co-experimentar la situación del otro yo. A pesar del autor no negar las retribuciones al campo sanitario, su planteamiento incorpora cinco puntos litigiosos que ameritan análisis (50). Breithapt refiere que "los conflictos sociales funcionarían como un poderoso disparador, pudiendo ser un desencadenante común de la empatía unilateral que puede llevar a la adopción de opiniones negativas sobre la otra parte", lo que acaba por llamar de 'efectos polarizadores de la empatía' (50). Más aún, su pensamiento alberga un lado 'egoísta', ya que "la suposición de que la empatía beneficia a su objetivo podría haber llevado a la gente a descuidar la parte que se beneficia más directamente, a saber, el propio empatizante" (50). El 'vampirismo empático' es introducido por Breithaupt de modo en que "el empatizante siente y experimenta el mundo vicariamente a través de otros y por lo tanto participa en su destino, sin tener sus mejores intereses en mente" (50). Otra compresión distorsionada de la práctica empática figura al involucrar actitudes sádicas en la asistencia geriátricagerontológica:

Sadismo empático, o crueldad empática en su forma básica, significa que un observador empático disfruta del dolor o sufrimiento de otro. Los sentimientos negativos de otro se traducen de alguna manera por el empatizante en sentimientos positivos por él o ella misma. La empatía sádica a menudo incluye la manipulación de los demás. La empatía manipuladora debe entenderse como una situación para el otro con el objetivo de hacer su respuesta emocional a esa situación inteligible, y por lo tanto posible para compartir (Traducción libre del inglés, $p$. 171).

En su último foco, Breithaupt discrimina la 'empatía humanitaria' como realmente siendo un tipo de 'empatía filtrada' (50). Admitiendo que "los seres se ayudan mutuamente en situaciones de necesidad, y la empatía es a menudo nombrada como el probable motivador de este comportamiento", por eso, explica que "la identificación con el ayudante todavía implica cierta empatía con la persona necesitada, pero a través de la figura del ayudante como medio o filtro". En otras palabras, "la persona necesitada sólo es vista como una persona necesitada, como alguien que es una persona en la medida en que él o ella motiva la acción del ayudante" (50). 
En su tesis 'The good, the bad and the necessity of empathy in ethics', Loftus (2019) remite la necesidad de entrelazar el debate ético con la utilidad empática [prisma multidimensional] desde las perspectivas sociológica y antropológica (51), para lo cual selecciona el caso de los individuos con inclinación sociópata. En dichos personajes, "la empatía parece causar un prejuicio y una derogación del respeto a sí mismo, pero también actúa como un poderoso motivador para la acción orientada a otros y proporciona información éticamente valiosa sobre los estados mentales". Por otro lado, llama la atención el modo de aproximación 'autista' postulada por Loftus (51) para los casos en que los individuos <retomemos los profesionales sanitarios> encuentran serias trabas intrínsecas y/o extrínsecas, para elucidar la práctica empática, que van desde el punto neurobiológico hasta los productos socio-antropológicos.

Songhorian (2019) se preocupa por investigar el rol de la moralidad en la práctica empática. Para la autora, el concepto atribuido coloquialmente a la empatía es falible y no se torna provechoso en el campo de la investigación interdisciplinar. Como justificativa, expone que la empatía, por sí misma, puede ser fácilmente influenciada por factores que no son moralmente relevantes (color de piel, género, nacionalidad, apariencias, etc.) y puede desencadenar formas injustificables de preferencias frente a algunos individuos, por ser similares o distantes al agente <empatizador> (52). Songhorian también discute el sadismo empático de modo similar a Breithaupt (50), pero toma como base teórica la vertiente alemana [Freude]- disfrutar del dolor y la desdicha del otro yo, cogitando que "un sádico no tiene una deficiencia neural o una activación reducida de lo que se puede llamar el 'circuito empático', sino que el trastorno debe encontrarse en un nivel diferente" (52).

Queda claro que los trabajos de Breithaupt (50), Loftus (51) e Songhorian (52) demuestran marcado interés por el ramo de la empatía moral, reconocido como un cuarto dominio en el campo de investigación empática. Esa, por su vez, recibió grandes aportes en los escritos de Morse et al., (1992), que retractaron que el empleo de la práctica empática terapéutica:

Puede ser apropiada en algunas interacciones entre enfermeras y pacientes; sin embargo, es necesario determinar cuándo debe utilizarse en los entornos clínicos, las formas en que se comunica la empatía emocional y moral entre las enfermeras y los pacientes, y los resultados (tanto positivos como negativos) que se obtienen cuando la enfermera utiliza la empatía terapéutica $(53$, p. 278).

Aunque son varios los instrumentos propuestos para sopesar actitudes empáticas entre profesionales y usuarios, los más citados internacionalmente <incluyendo al IRI, elaborado por Davis (16)> presentan un problema común: sus preguntas no son relevantes para las profesiones sanitarias porque no fueron desarrolladas específicamente para tales fines. Recientemente, un estudio de tipo revisión sistemática consiguió identificar los once instrumentos más utilizados por los profesionales sanitarios y advirtió que "ninguno se desarrolló específicamente para medir la empatía de los profesionales de la atención geriátrica" (54), indicando la existencia de una brecha científico-práctica en la clínica geriátrica-gerontológica.

La adquisición de experiencias empáticas aún durante la formación de pregrado y grado en las carreras sanitarias es más una excepción a la regla de que una actividad institucionalizada en los centros de educación superior (55, 56). Tal carestía de conocimiento aplicado revela un tipo de desacato en la atención integral al geronte, pues para su consecución es imperativo que haya una articulación entre los sistemas públicos de salud y educación. En la misma medida, Costa y colaboradores (2009) orientan que "al 
acoger a la población, las unidades de salud prestan servicios de calidad y resolutividad, y sus acciones son integrales cuando atienden las necesidades y demandas de la población" (57), al tiempo en que "la atención debe ser condicionante para la orientación de los servicios y prestaciones sanitarias desde una atención interdisciplinaria" (57).

Para realmente ejercitar el acto empático asertivo, ambas partes precisan desarrollar un alto nivel de Inteligencia Emocional (IE) y esta, infelizmente, parece ser muy deficiente en los profesionales sanitarios que atienden adultos mayores (58). Así, la IE sería una habilidad genuina para sentir emociones en respuesta al otro yo, ya que tiene que ver con la construcción de puentes y alianzas, y ser capaz de repararlos si estos se llegaran a dañar (59). Por parecido, la IE suele caracterizarse como una capacidad cognitiva que implica el procesamiento cognitivo de la información emocional (60). En lo que concierne a los profesionales sanitarios, una elevada IE brindaría la capacidad para encontrar salidas íntegras y sensibilizadas, en contextos de angustia y sufrimiento para el adulto mayor.

En Gardner (1987), la cuestión de la inteligencia es vista más allá de las fronteras del conocimiento adquirido. Sus postulaciones parten del reconocimiento de que la brillantez académica no lo es todo, para lo que propone un modelo de 'Inteligencias Múltiples': lingüístico-verbal, lógico-matemática, musical, espacial, científico-corporal, interpersonal, intrapersonal y naturalistica. Observando el caso específico de las inteligencias inter e intrapersonal, éstas "conforman la IE y juntas determinan nuestra capacidad de dirigir nuestra propia vida de manera satisfactoria" (61, p.4). El argumento basilar de Gardner (1998) supone que "la inteligencia interpersonal permite comprender y trabajar con los demás; la inteligencia intrapersonal permite comprenderse y trabajar con uno mismo" (62, p.10).

Recientemente, estudio portugués, que tuvo por objetivo evaluar el nivel de inteligencia emocional de los adultos mayores y verificar si variables independientes interferían en sus dimensiones, permitió comprobar que el desarrollo emocional en las personas adultas mayores permite identificar y minimizar las situaciones de tristeza, aislamiento social y depresión que interferirán en la calidad de vida personal y social (63). Otro ejemplo investigativo sobre IE en adultos mayores se registra en el trabajo de Silva (2015), en el cual se destacan cuatro esferas que merecen destaque. La 'percepción emocional', definida como la capacidad del individuo de reconocer emociones propias y ajenas. La 'emoción como facilitadora del pensamiento', admitiendo que los procesos cognitivos pueden sufrir influencias por las emociones del individuo. La 'comprensión emocional', que considera tres elementos principales: identificación y codificación de las emociones, significados y origen de la relación. Y, por último, la 'gestión emocional', que sería la capacidad del individuo de regular sus emociones, sobreponiendo resultados positivos sobre los negativos (64, p.10).

Sobre otro foco aprensivo, cabe señalar que el fomento de vivencias pragmáticas, a lo largo del desarrollo académico-profesional, presupone una respuesta inmediata a la misión de formar futuros profesionales que se preparan para actuar en la clínica geriátricagerontológica, de manera competente, con las habilidades y actitudes apropiadas para trabajar con adultos mayores $(45,47,65)$. Subsiguientemente, la construcción e incorporación de prácticas complementares al campo clínico geriátrico (por ejemplo: simulación de problemas sensoriales, cognitivos y de movilidad) podría servir como herramienta recurrente para la formación de profesionales sanitarios plenamente instruidos para lidiar con los apuros de la atención integral a la salud del adulto mayor (66), tanto en los casos asistenciales $(67,68,69)$, como en la comprensión holística de los aspectos socio-sanitarios vinculados al proceso de envejecimiento humano $(70,71)$. 


\section{Conclusiones}

- El concepto de empatía ha demostrado innúmeras variaciones a lo largo de la historia. Sus propulsores en el campo sanitario, basados en sus áreas primarias del conocimiento, si bien han mantenido el consenso de ésta ser una habilidad social que necesita ser aprendida, se han encargado de reiterar que su uso no debe ser extremado, ni al punto positivo, ni al punto negativo, constatando la eficacia del distanciamiento terapéutico.

- La institucionalización de la práctica empática en los servicios de salud propicia oportunidades para la formación del vínculo asistencial entre el profesional sanitario y el adulto mayor, por consiguiente, una asistencia empática abriría caminos para la promoción de la atención integral a la salud del adulto mayor aspirada por los sistemas públicos de salud.

- Además de combatir posicionamientos apáticos, la pretensión de incorporar la práctica empática en los servicios sanitarios tiene como gran enemigo la inexistencia de instrumentos específicos para medir atributos geriátricos y gerontológicos, factor que impide a los profesionales sanitarios amplificar las características y necesidades propias del geronte. En contrapartida, se hace necesario que los profesionales sanitarios encuentren momentos de aprendizaje continuado y permanente, con el objetivo de promover contextos reales que pongan a prueba su capacidad de respuesta empática.

- La readecuación y actualización de los currículos académicos juega un papel esencial, puesto que aprecia la relevancia de formar profesionales sanitarios conscientes de las rápidas mudanzas del segmento poblacional de mayor edad y la propia heterogeneidad del proceso de envejecimiento humano.

Material suplementario: No hay.

Financiación: Ministerio de Educación, Brasil (MEC) y Ministerio de Salud, Brasil (MS). Auxilio financiero otorgado a Gutiérrez-Murillo RS, en el marco de investigación científica-profesional del Programa de Residencia Multiprofesional en Salud Familiar - Universidad Federal de la Integración Latinoamericana (20202022).

Declaración de conflicto of interés: Los autores declaran no tener ningún conflicto de intereses.

\section{Referencias}

1. Pham TN, Vo DH. Aging population and economic growth in developing countries: a quantile regression approach. Emerging Markets, Finance and Trade. 2019;(1):1-15. DOI: https:/ / doi.org/10.1080/1540496X.2019.1698418.

2. World Health Organization. Global status report on noncommunicable diseases 2014: attaining the nine global noncommunicable diseases target; a shared responsibility. Recuperado de: https://apps.who.int/iris/bitstream/handle/10665/148114/9789241564854_eng.pdf;jsessionid=75C70E CC5348AF8848118BBCB9C7DCAB? sequence=1.

3. Oliveira MR, Veras Peixoto R, Cordeiro Albuquerque H. A importância da porta de entrada no sistema: o modelo integral de cuidado para o idoso. Physis: Revista de Saúde Coletiva. 2018;28(4):1-24. DOI: https://doi.org/10.1590/s0103-73312018280411

4. Freitas MPD, Moraes EN. Estrutura da rede de atenção à saúde da pessoa idosa. In: Borges APA; Coimbra AMC (Org.). Envelhecimento e saúde da pessoa idosa. Rio de Janeiro, RJ: EAD, ENSP, 2008. 340p. Recuperado de: http://www5.ensp.fiocruz.br/biblioteca/dados/txt_594481029.pdf.

5. Merhy EE. O ato de cuidar: a alma dos serviços de saúde. São Paulo: Departamento de Medicina $\begin{array}{lllll}\text { Preventiva } & \text { e } & \text { Social. } & \text { Recuperado }\end{array}$ https://www.nescon.medicina.ufmg.br/biblioteca/imagem/2103.pdf.

6. Mattos CW, Derech RD. Palliative care provided by family and community doctors in primary health care in Brazil: a national survey. Revista Brasileira de Medicina de Família e Comunidade. 2020;15(42):1-10. DOI: https:/ / doi.org/10.5712/rbmfc15(42)2094. 
7. Azevedo CR. Instrumentos de avaliação da empatia: uma revisão sistemática da literatura (Monografia de Especialização em Psicologia - ênfase em avaliação psicológica). Universidade Federal do Rio Grande do Sul. 2014; 1-30. Recuperado de: https:/ / www.lume.ufrgs.br/handle/10183/102339.

8. Pérez AC, Nayely K, León Q, Coronado EAG. Empatía, comunicación asertiva y seguimiento de normas: un programa para desarrollar habilidades para la vida. Enseñanza e Investigación en Psicología. 2017;22(1):58-65. Recuperado de: http:/ / www.redalyc.org/articulo.oa?id=29251161005.

9. Universidad de Salamanca. Dicciomed: Diccionario médico-biológico, histórico y etimológico. 'empatía'. https://dicciomed.usal.es/palabra/empatia

10. Wispé L. The distinction between sympathy and empathy: to call forth a concept a word is needed. Journal of Personality and Social Psychology. 1896;50(2):314-321. DOI: https://doi.org/10.1037/00223514.50.2.314.

11. Pires MFDN, Roazzi A. Empatia e sua avaliação: considerações teóricas e metodológicas. Revista Amazônica. $\quad 2016 ; \quad 17(1): \quad 158-172$. https://www.researchgate.net/publication/313038718_Empatia_e_sua_avaliacao_Consideracoes_teorica s_e_metodologicas

12. San Martín AZ, Ortega-Sánchez D. Empatía, empatía histórica y empatía prehistórica: una aproximación conceptual desde la enseñanza de las ciencias sociales. Didáctica de las Ciencias Experimentales y Sociales. 2020;38(1):3-16. DOI: https://doi.org/10.7203/DCES.38.15648.

13. Markovsky B, Berger SM. Crowd sound and mimicry. Personality and Social Psychology Bulletin. 1893;9(1):90-6. DOI: https://doi.org/10.1177/0146167283091012.

14. Kariaginaand TD, Ivanova AV. Empathy as an ability: structure and development during training in psychological counselling. Journal of Russian and East Psychology. 2017;54(6):470-97. DOI: https:/ /doi.org/ $\underline{10.1080 / 10610405.2017 .1448180 .}$.

15. Davis MH. A multidimensional approach to individual difference in empathy. JSAS Catalog of Selected Documents in Psychology. 1980, 10, p.85.

16. Davis MH. Measuring individual differences in empathy: evidence for a multidimensional approach. Journal of Personality and Social Psychology. 1983;44(1):113.26. DOI: https://doi.org/10.1037/00223514.44.1.113.

17. Svenaeus F. Edith Stein's phenomenology of sensual and emotional empathy. Phenomenology Cognition Science. 2018;17(1):741-60. DOI: https:/ / doi.org/10.1007/s11097-017-9544-9.

18. Toro-López JR, Castro MG, Molini JAT, et al. Importancia de la empatía en el estado de salud. HYGIA. 2020;103(1):10-7. Recuperado de: https:/ / www.researchgate.net/publication/338558937.

19. Rueckert L, Naybar N. Gender differences in empathy: the role of the right hemisphere. Brain and Cognition. 2008;67(1):162-7. DOI: https://doi.org/10.1016/j.bandc.2008.01.002.

20. Vemuni K, Surampudi BR. Evidence of stimulus correlated empathy modes - Groups ICA of fMRI data. Brain and Cognition. 2015;15(1):32-43. DOI: https://doi.org/10.1016/j.bandc.2014.12.006.

21. Chismar D. Empathy and sympathy: the important difference. The Journal of Value Inquiry. 1988;22(4):25766. DOI: https://doi.org/10.1007/bf00136928.

22. Svenaeus F. The relationship between empathy and sympathy in good health care. Medicine, Health Care and Philosophy. 2015;18(1):267-77. DOI: https://doi.org/10.1007/s11019-014-9801-x.

23. Tobón DJ. Empathy and sympathy: two contemporary models of character engagement. In: The Palgrave Handbook of the Philosophy of Film and Motion Pictures. Carroll N, Di Summa LT, Loht S (Ends). 2019. 1047 pp.

24. Mosquera M. Comunicación en salud: conceptos, teorías y experiencias. Comminit. La iniciativa de la comunicación, 2003.

25. Hernández IR. Comunicación en salud: conceptos y modelos teóricos. Perspectivas de la comunicación. 2011;4(1):127-40.

https://www.researchgate.net/publication/277273859 Comunicacion en salud Conceptos y modelos teoricos.

26. Muñiz MJI. Relaciones interpersonales y calidad de vida: programa de competencia social y comunicación en personas mayores de 60 años. Trabajo de Fin de Máster en Psicopedagogía. Universidad de Valladolid. 2018. 98p. Recuperado de: http:/ / uvadoc.uva.es/handle/10324/32802. 
27. Bermejo JC. Empatía terapéutica. La compasión del sanador herido. Bilbao: Desclée de Brouwer. 2012. Recuperado https://ache-counselling.org/wp-content/uploads/2014/03/Empatia terapeutica y su aplicacion en s alud.pdf.

28. Guedes MBOG, Lima KC, Caldas $\mathrm{CP}$, et al. Apoio social e o cuidado integral à saúde do idoso. Physis Revista de Saúde Coletiva. 2017;27(4):1185-1204. DOI: http:/ /dx.doi.org/10.1590/S0103-73312017000400017.

29. Medeiros KKSI, Júnior EPP, Bousquat A, et al. The challenge of integrality in elderly care in the scope of primary health care. Saúde e Debate. 2017;41(3):288-94. DOI: https:/ / doi.org/10.1590/0103-11042017S322.

30. Seixas CT, Baduy RS, Cruz KT, et al. The power of the bond for healthcare production: what guiding users teach us. Interface (Botucatu). 23:e170627. DOI: https://doi.org/10.1590/Interface.170627.

31. Yuguero O, Esquerda M, Viñas J, et al. Ética y empatía: relación entre razonamiento moral, sensibilidad ética y empatía en estudiantes de medicina. Revista Clínica Española. 2019(2):73-8. DOI: https://doi.org/10.1016/j.rce.2018.09.002.

32. García MCH. Fatiga por compasión entre profesionales sanitarios de oncología y cuidados paliativos. Psicooncología. 2017;14(1):53-70. DOI: http://dx.doi.org/10.5209/PSIC.55811.

33. Albaladejo IM. Fatiga por compasión en profesional sanitario especializado en cuidados paliativos. Trabajo Final de Máster en Prevención de Riesgos Laborales. Universidad Miguel Hernández. 2019. 54p. Recuperado de: http://193.147.134.18/bitstream/11000/5694/1/MARTINEZ\%20ALBALADEJO\%2C \%20INMACULADA\%20TFM.pdf.

34. Carrió FB. Simpatía-empatía-compasión: parecen lo mismo pero no lo son. Folia Humanística - Revista de Salud, Ciencias Sociales y Humanidades. 2018;10(1):1-17. DOI: http:/ / dox.doi.org/10.30860/0042.

35. Vendrell I. Simpatía, empatía y otros actos sociales: las raízes olvidadas de la sociología fenomenológica. Espacio Abierto Cuaderno Venezolano de Sociología. 2008;17(2):303-15. Recuperado de: https://www.redalyc.org/pdf/122/12217206.pdf.

36. Pintado S. Programas basados en mindfulness para el tratamiento de la fatiga por compasión en personal sanitario: una revisión. Terapia Psicológica. 2018;36(2):71-80. DOI: http://dx.doi.org/10.4067/S0718$\underline{48082018000200071 .}$.

37. Teresa R, Reis-Queiroz J, Hoga LAK. A importância da empatia no cuidado em saúde e enfermagem. Revista Brasileira de Enfermagem REBEn. 2017;70(3):697-8. DOI: http:/ / dx.doi.org/10.1590/0034-7167-2016$\underline{0032 .}$

38. Monteiro B, Queirós C, Marques A. Empatia e engagement como preditores do burnout em cuidadores formais de idosos. Psicologia, Saúde $\mathcal{E}$ Doenças. 2014;15(1):1-12. DOI: http://dx.doi.org/10.15309/14psd150102.

39. Araújo JP, Costa YMN. Escala Analógica de Empatia Emocional para avaliação da percepção da depressão em idosos. Trabalho de Conclusão de Curso (Graduação em Medicina) - Universidade Federal de Campina Grande, Cajazeiras - 2016. Recuperado de: http:/ /dspace.sti.ufcg.edu.br:8080/jspui/handle/riufcg/7747.

40. Gholamzadeh S, Khastavaneh M, Khademian Z, et al. The effects of empathy skills on nursing students' empathy and attitudes toward elderly people. BMC Medical Education. 2018;198(18):1-7. DOI: https://doi.org/10.1186/s12909-018-1297-9.

41. Hannan J, Sanchez G, Musser, et al. Role of empathy in the perception of medical errors in patient encounters: a preliminary study. BMC Res Notes. 2019;327(12):1-5. DOI: https://doi.org/10.1186/s13104019-4365-2.

42. Longhi A. L'abuso nell'assistenza agli anziani in rapporto con i livelli di empatia del personale di assistenza: studo osservazionale. Tesi di Laurea (Corso di Laurea in Infermieristica). Università delig Studi di Padova. Recuperado de: http://tesi.cab.unipd.it/51026/.

43. Fuentes CLC. La influencia del apoyo social en el estado emocional y las actitudes hacia la vejez y el envejecimiento en una nuestra de ancianos. International Journal of Psychology and Psychological Therapy. 2014;14(3):365-377. Recuperado de: http:/ / www.redalyc.org/articulo.oa?id=56032544004.

44. García-Vera EM, Robles-Rodríguez A, Villegas-Bernabé ML, et al. La comunicación médico-paciente: ¿reto para el paciente geriátrico o para el médico familiar? Atención Familiar. 23(2):63-66. DOI: https://doi.org/10.1016/j.af.2016.03.007. 
45. Pison MR, Yáñez NV, Fritis LM, et al. Empatía y estudiantes de kinesiología. Revista de Ciencias Médicas de Pilar del Río. 2019;23(2):295-301. Recuperado de: http://revcmpinar.sld.cu/index.php/publicaciones/article/view/3863.

46. Morales-Concha L, Ccarita-Yucra K, Marroquin-Santa Cruz JA, et al. Evaluación de la empatía en estudiantes de medicina humana en una universidad pública de la sierra sur del Perú. Educación Médica. 2017;19(6):327-32. DOI: https:/ / doi.org/10.1016/j.edumed.2017.04.006.

47. San-Martín M, Roig-Carrera H, Villalonga-Vadell RM, et al. Empatía, habilidades de colaboración interprofesional y aprendizaje médico permanente en residentes españoles y latinoamericanos que inician los programas de formación médica especializada en España: resultados preliminares. Atención Primaria. 2017;49(1):6-12. DOI: http://dx.doi.org/10.1016/j.aprim.2016.02.007.

48. Díaz-Martínez LA, Armest MHC, Rojas MJD. La formación médica en comunicación de malas noticias: una revisión narrativa. Rev Esp Edu Med. 2020;1(2):32-42. DOI: https://doi.org/10.6018/edumed.439731.

49. Martínez-Gonzáles A, Sánchez-Mendiola M, Olivares-Olivares SL, et al. Colaboración de tres escuelas de medicina de México en un examen clínico objetivo estructurado (ECOE). Investigación en Educación Médica. 2020;9(36):58-69. DOI: https:/ / doi.org/10.22201/fm.20075057e.2020.36.20258.

50. Breithaupt F. The bad things we do because of empathy. Interdisciplinary Science Review. 2018;43(2):166-74. DOI: https://doi.org/10.1080/03080188.2018.1450928.

51. Loftus E. The good, the bad and the necessity of empathy in ethics. Scripps Senior Thesis. 2019;1350, 50p. Recuperado de: https://scholarship.claremont.edu/scripps theses/1399.

52. Songhorian S. The contribution of empathy to ethics. International Journal of Philosophical Studies. 2019;27(2):244-64. DOI: https://doi.org/10.1080/09672559.2019.1619266.

53. Morse JM, Anderson G, Bottorf JL, et al. Exploring empathy: a conceptual fit for nursing practice? Image: The Journal of Nursing Scholarship. 1992;24(4):273-80. DOI: https://doi.org/10.1111/j.15475069.1992.tb00733.x.

54. Hong H \& Han A. A systematic review on empathy measurement tools for care professionals. Educational Gerontology. 2020;46(2):72-83. DOI: https://doi.org/10.1080/03601277.2020.1712058.

55. Varkey P, Chutka DS, Lesnick TG. The aging game: improving medical students' attitudes toward caring for the elderly. Journal of the American Medical Directors Association. 2006;7(4):224-29. DOI: https://doi.org/10.1016/j.jamda.2005.07.009.

56. Altay D. Developing empathy towards older adults in design. Educational Gerontology. 2017;43(4):198-208. DOI: http://dx.doi.org/10.1080/03601277.2016.1273733.

57. Costa MFBN, Ciosak SI, Bartolomé AG, et al. La integralidad en la atención primaria de salud en los sistemas de salud brasileño y español. Aten Primaria. 2009;41(7):402-404. DOI: https://doi.org/10.1016/j.aprim.2009.02.010.

58. Nightingale S, Spiby H, Sheen K, et al. The impact of emotional intelligence in health care professionals on caring behavior towards patients in clinical and long-term care settings: findings from an integrative review. International Journal of Nursing Studies. 2018;80(1):106-117. DOI: https://doi.org/10.1016/j.ijnurstu.2018.01.006.

59. Fernandez CSP. Emotional Intelligence in the workplace. J Public Health Management Practice. 2007;13(1):80-2. DOI: https://doi.org/10.1097/00124784-200701000-00013.

60. Qualter P, Gardner KJ \& Whiteley HE. (2007). Emotional intelligence: review of research and educational implications. Pastoral Care in Education, 25(1):11-20. DOI: https://doi.org/10.1111/j.14680122.2007.00395.x

61. Gardner H. (1987). La teoría de las inteligencias múltiples. Santiago de Chile: Instituto Construir, 287-305.

62. Gardner H. (1998). Inteligencias múltiples: la teoría en la práctica. Barcelona: Paidós, 1-14.

63. Andrade A, Duarte J, Cruz C et al. (2019). Inteligência emocional em idosos portugueses. INFAD Revista de Psicologia, 4(1):331-38. DOI: https:/ / doi.org/10.17060/ijodaep.2019.n1.v4.1566.

64. Silva DCM. (2015). Inteligência emocional e estilos de coping: estudo exploratório numa população idosa. Dissertação (Mestrado em Gerontologia Social). Instituto Superior de Serviço Social do Porto. 58p. Recuperado de: http://comum.rcaap.pt/bitstream/10400.26/10909/1/Diana\%20Catarina\%20Martins \%20da\%20Silva.pdf. 
65. Henry BW, Ozier AD. Empathetic responses and attitudes about older adults: how experience with aging game measures up. Educational Gerontology. 2011;37(1):924-41. DOI: https://doi.org/10.1080/03601277.2010.495540.

66. Hubbard RW \& Santos JF. Empathy training as an instructional tool for geriatric health professionals. Educational Gerontology. 1981;6(3):191-4. DOI: http://dx.doi.org/10.1080/0380127810060209.

67. Langer N. Integrating compliance, communication and culture: delivering health care to an aging $\begin{array}{llll}\text { population. } & \text { Educational } & \text { Gerontology. }\end{array}$ http://dx.doi.org/10.1080/03601270701838381.

68. Galanos AN, Cohen HJ \& Jackson TW. Medical education in geriatrics: the lasting impact of the aging game. Educational Gerontology. 1993;19(7):675-82. DOI: http://dx.doi.org/10.1080/0360127930190708.

69. Gholamzadeh S, Khastavaneh M, Khademian Z, et al. The effects of empathy skills training on nursing students' empathy and attitudes toward elderly people. BCM Medical Education. 2018;18(198):1-7. DOI: https://doi.org/10.1186/s12909-018-1297-9.

70. Langer N \& Ribarich M. Using narratives in healthcare communication. Educational Gerontology. 2008;35(1):55-62. DOI: http:/ / dx.doi.org/10.1080/03601270802388930.

71. Cherix K \& Coelho JNE. The care of elderly as a field of inter-subjective relations: ethic reflections. Interface (Botucatu). 2017; 21(62):579-88. DOI: https:/ / doi.org/10.1590/1807-57622015.0492.

(C) 2021 por los autores. Enviado para su publicación en acceso abierto bajo los términos y condiciones de la licencia Creative Commons Attribution (CC BY) (http://creativecommons.org/licenses/by/4.0/). 